\title{
Nine Years of VLBI Imaging of Supernova 1993J
}

M. F. Bietenholz ${ }^{1}$, N. Bartel ${ }^{1}$, M. P. Rupen ${ }^{2}$, A. J. Beasley ${ }^{3}$,

D. A. Graham ${ }^{3}$, Altunin, V. I. ${ }^{4}$, T. Venturi ${ }^{5}$, G. Umana ${ }^{5}$,

W. H. Cannon ${ }^{6}$, and J. E. Conway ${ }^{8}$

1 York University, Canada

2 National Radio Astronomy Observatory, USA

3 CARMA, Owens Valley Radio Observatory, USA

4 Max-Planck-Institut für Radioastronomie, Germany

5 Jet Propulsion Laboratory, Caltech, USA

${ }^{6}$ Instituto di Radioastonomia, CNR, Italy

7 York University \& Space Geodynamics Lab/CRESTech, Canada

${ }^{8}$ Onsala Space Observatory, Sweden

Summary. We present a selection of our VLBI images of SN 1993J, along with our latest results on its expansion and its shell structure.

\section{Introduction}

Supernova 1993J is the most comprehensively monitored radio supernova to date. Our extensive campaign of VLBI observations [1, 2, 3, 4], at 35 epochs, at frequencies between 1.7 and $22 \mathrm{GHz}$, using up to 18 antennas in a global array, has allowed us to make images from $30 \mathrm{~d}$ to $9 \mathrm{yr}$ after the explosion (see [5] for parallel observations). Our images are all phase-referenced to the stationary core, M81*ore, of the nuclear source in the host galaxy, M81 [3], which allows us to display them relative to the supernova's explosion center.

\section{Model Fitting}

At every epoch, we fit a spherical shell model directly to the $u$ - $v$ data in order to estimate the position of the geometrical center, the size, and for later epochs, the shell thickness of SN 1993J. We determined the projected motion of the center of SN 1993J relative to $\mathrm{M} 81_{\text {core }}^{*}$ to be $<480 \mathrm{~km} \mathrm{~s}^{-1}$, which is much smaller than the expansion velocities of $17,200 \mathrm{~km} \mathrm{~s}^{-1}$ at $t=30 \mathrm{~d}$ and $8900 \mathrm{~km} \mathrm{~s}^{-1}$ at $t=1600 \mathrm{~d}$ after shock breakout (1993 Mar. 28: $t=0 \mathrm{~d})[2]$. SN 1993J is still very circular 9 years after the explosion, and its expansion from the explosion center is isotropic to within $5.5 \%$ [3].

We show the expansion curve of SN 1993J in Fig. 1 (see [6] for paralle] results). The expansion is more complex than a simple power-law. From almost free expansion at $t<300 \mathrm{~d}$, the deceleration grows until $t \sim 1600 \mathrm{~d}$, 


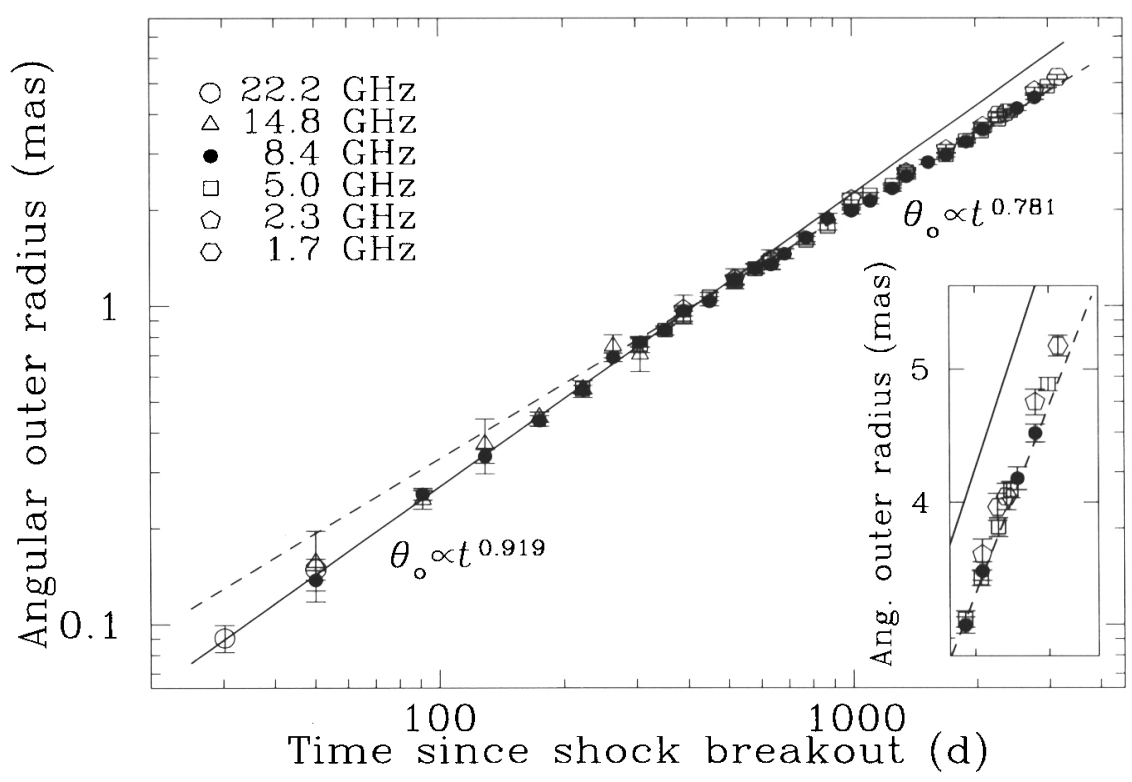

Fig. 1. The expansion curve of SN 1993J derived from 34 epochs of VLBI observations at frequencies between 1.7 and $22 \mathrm{GHz}$. The angular outer radii, $\theta_{0}$, are derived from a spherical shell model fit directly to the $u-v$ data. The inset shows outer radii for the latest epochs in more detail. The solid and dotted lines are powerlaw fits to the expansion, showing a change in the power-law index from 0.919 to 0.781 at $t \sim 600 \mathrm{~d}$. A further change in the index is visible in the inset (see [2] for more detail).

and then decreases again. For details and the interpretation of this behavior we refer the reader to [2].

\section{Images}

We present a selection of our images of SN 1993J at 8.4 and $5.0 \mathrm{GHz}$ in Fig. 2 (see [1, 4] for more images). The shell structure first becomes visible at $t=175 \mathrm{~d}$. Already at this stage the brightness was modulated around the ridge, with a distinct minimum to the west and a maximum to the eastsouth-east. Over the next $\sim 300 \mathrm{~d}$, this pattern appears to rotate, and by $t=451 \mathrm{~d}$ the maximum is to the south and the minimum to the north. The pattern of modulation then becomes more complex, with two hot spots appearing to the east and west by $t=774 \mathrm{~d}$, and a third in the south by $t=1107 \mathrm{~d}$. By the end of our observing period at $t=3345 \mathrm{~d}$, the opening in the north had become a hot spot and there were two further hot spots, to the south-southeast, and the west-southwest. At that time, the radius of SN 1993J was $\sim 12,000 \mathrm{AU}$. 
With the increasing relative angular resolution we were able to determine the shell thickness, again using model-fitting. The best fit model had a shell thickness of $25 \pm 3 \%$ of the shell's outer radius, and also had some absorption in the center of the shell. Models without absorption result in a thinner shell but also a poorer fit [4]. The best visual indication of the shell thickness is given by a composite image, also shown in Fig. 2, made from our last three 8.4 GHz data sets scaled and combined (see [4] for details). 

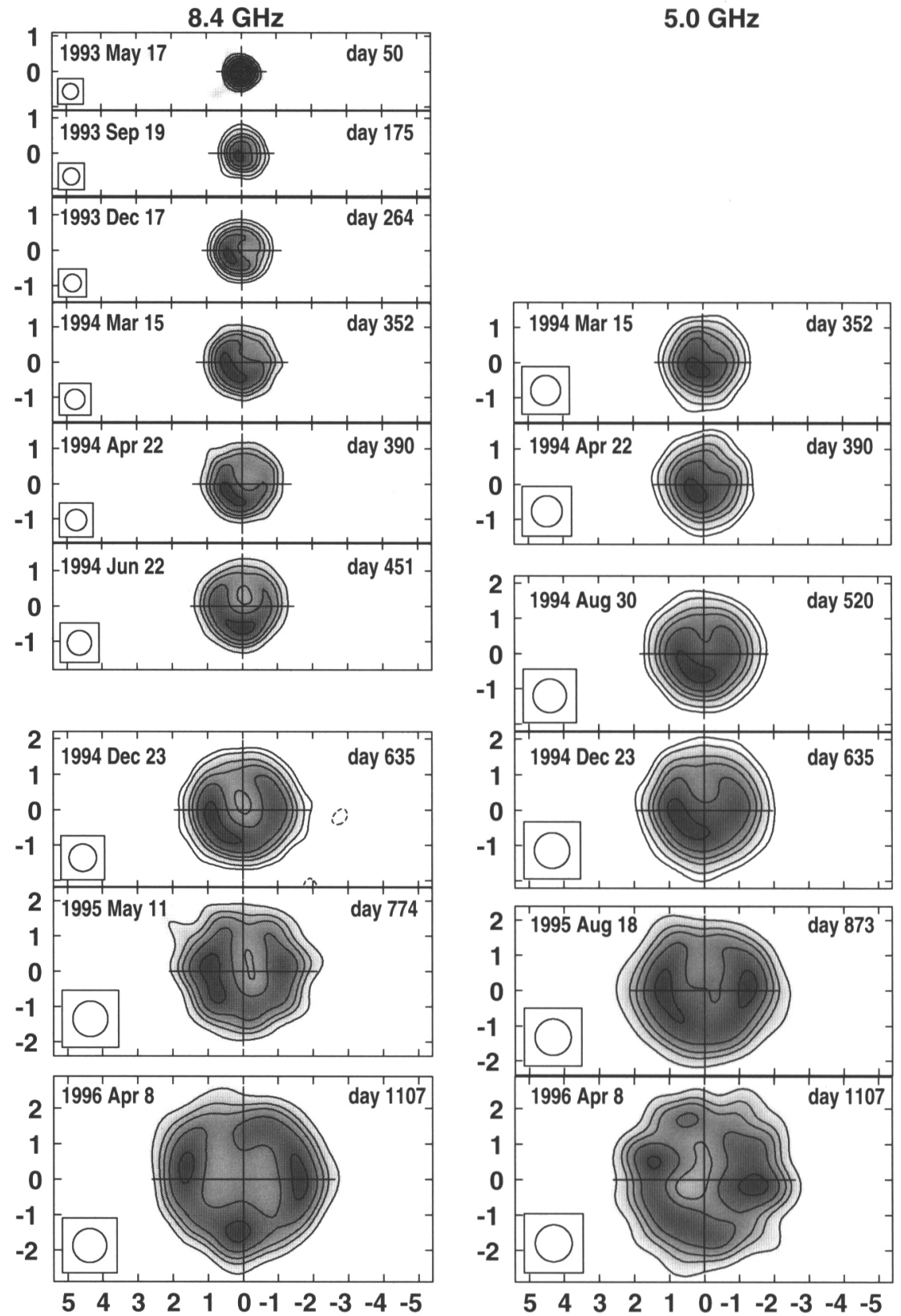


\subsection{GHz}
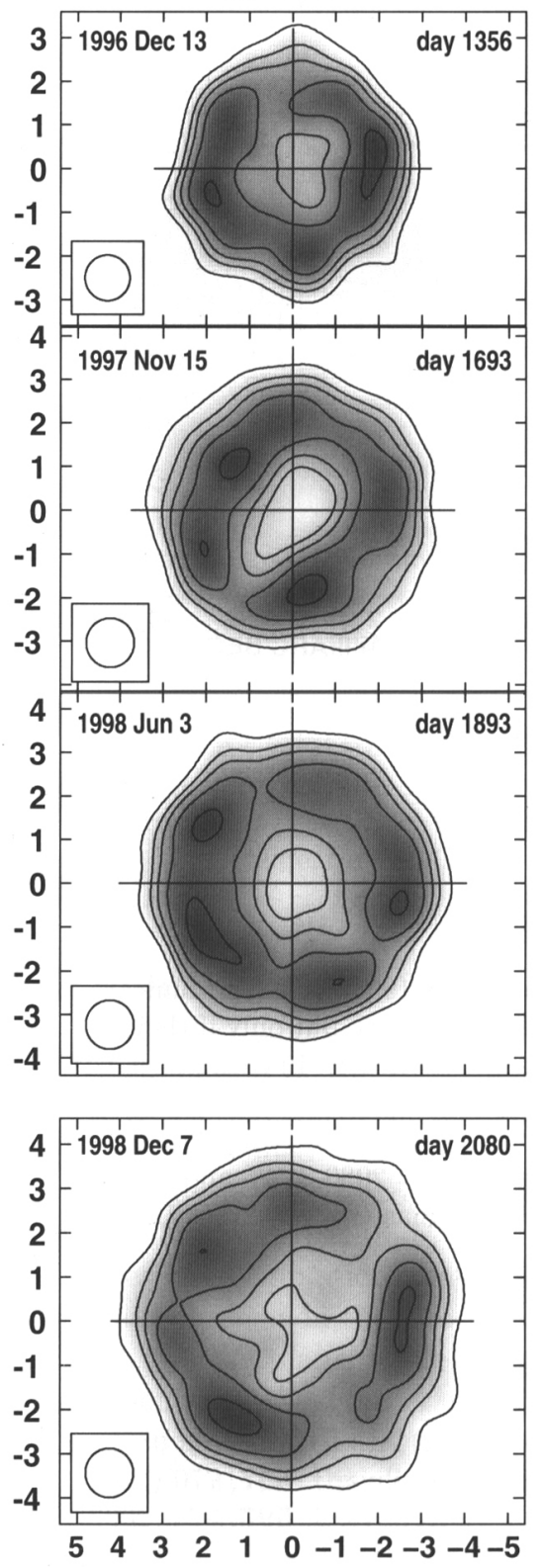

$5.0 \mathrm{GHz}$
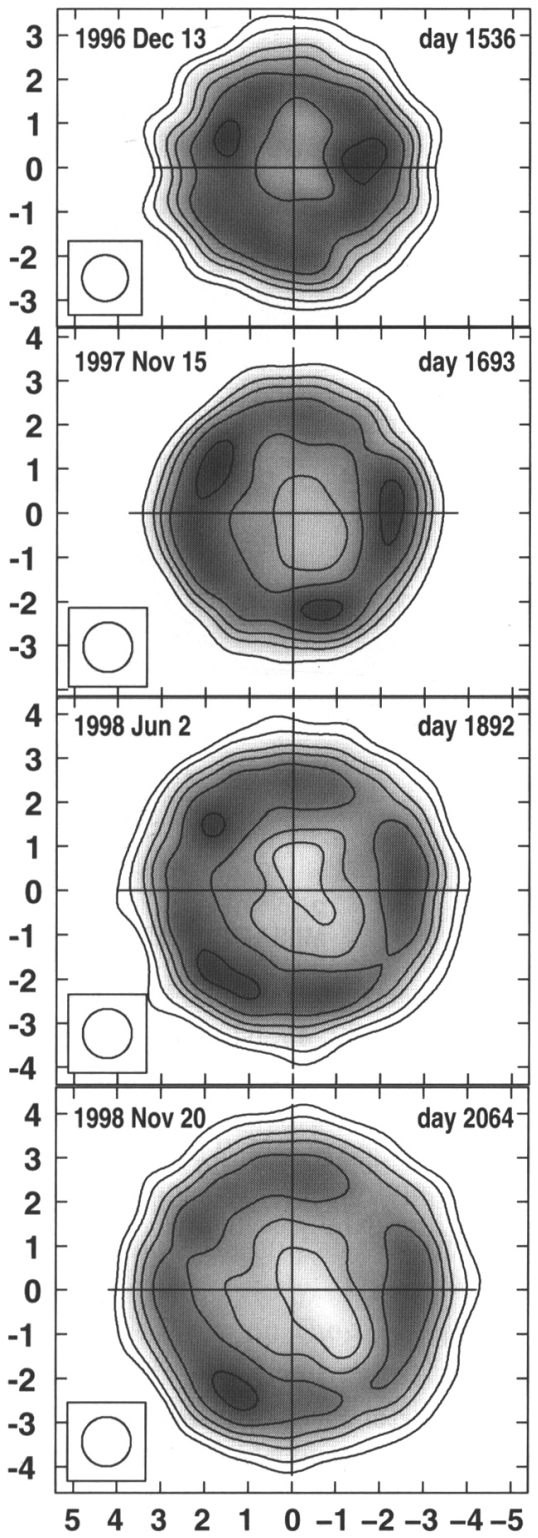


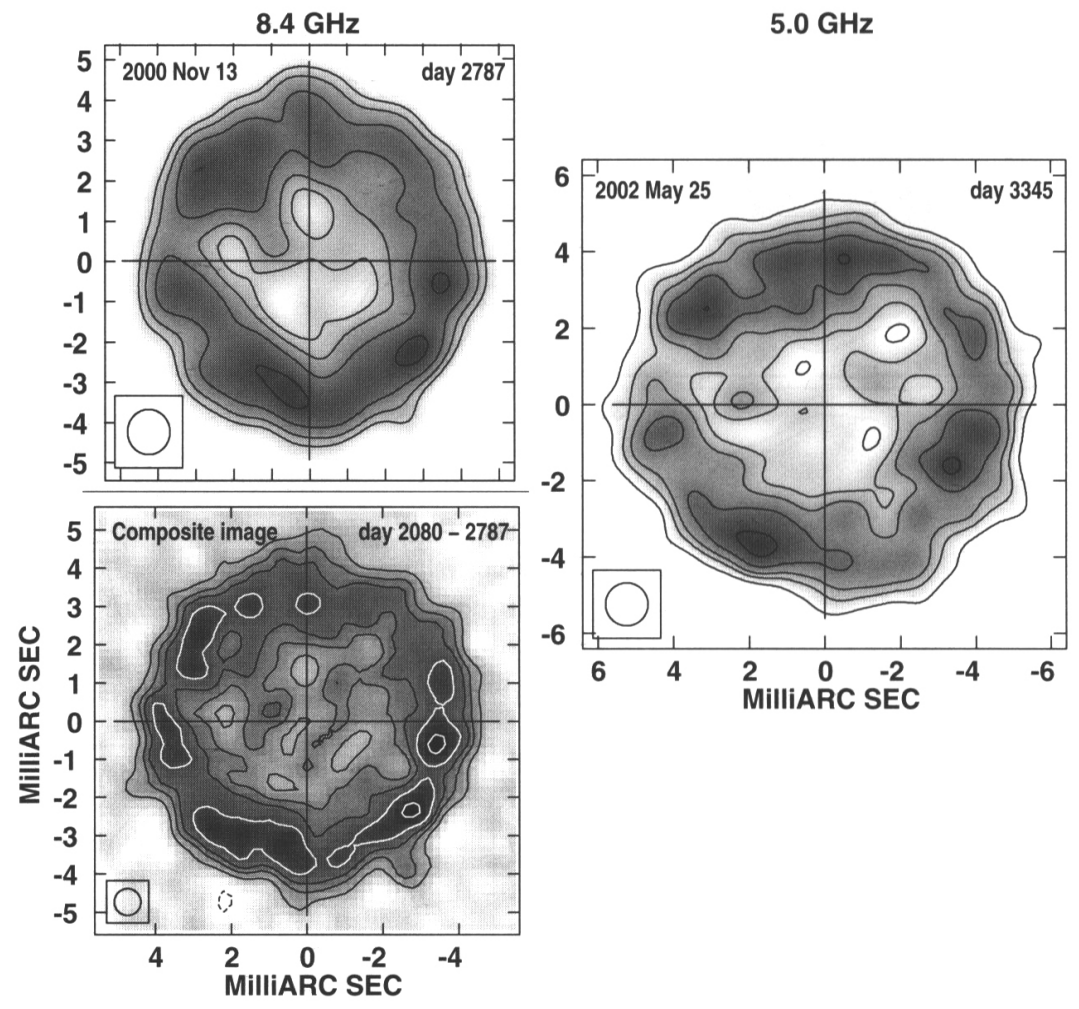

Fig. 2. Selected 8.4 and $5.0 \mathrm{GHz}$ radio images of SN 1993J. The contours are drawn at $1,2,4, \ldots, 32,45,64$, and $90 \%$ of the peak brightness, but only if they are $>3 \times$ the rms background noise. North is up and east is to the left. In each panel, the large cross indicates the position of the explosion center, and the resolution (FWHM) is indicated at lower left. The last $8.4 \mathrm{GHz}$ image is at higher resolution (0.7 mas), and is a composite image, made from the data at $t=2080,2525$ and $2787 \mathrm{~d}$ (1998 Dec. to 2000 Nov.), scaled in outer radius to 4.49 mas (i.e. $t=2787 \mathrm{~d}$ ).

\section{References}

1. N. Bartel et al. : Science 287, $112(2000)$

2. N. Bartel et al. : Astrophys. J. 581, 404 (2002)

3. M.F. Bietenholz, N. Bartel, M.P. Rupen: Astrophys. J. 557, 770 (2001)

4. M.F. Bietenholz, N. Bartel, M.P. Rupen: Astrophys. J. 597, 374 (2003)

5. J.M. Marcaide et al. : Astrophys. J. Lett. 486, L31 (1997)

6. J.M. Marcaide et al. : In: Proceedings, of the $6^{\text {th }}$ VLBI Network Symposium, 2002, ed. by E. Ros et al. pp. 239-242 